\title{
ORDINARY DIFFERENTIAL OPERATORS UNDER STIELTJES BOUNDARY CONDITIONS $\left({ }^{1}\right)$
}

\author{
BY \\ RICHARD C. BROWN AND ALLAN M. KRALL
}

\begin{abstract}
The operator $L_{p} y=y^{\prime}+P y$, whose domain is determined in part by the Stieltjes integral boundary condition $\int_{0}^{1} d v(t) y(t)=0$, is studied in $\mathcal{L}_{n}^{P}(0,1), 1 \leq p<\infty$. It is shown that $L_{p}$ has a dense domain; hence there exists a dual operator $L_{q}^{+}$operating on $\mathcal{L}_{n}^{q}(0,1)$. After finding $L_{q}^{+}$we show that both $L_{p}$ and $L_{q}^{+}$are Fredholm operators. This implies some elementary results concerning the spectrum and states of $L_{p}$. Finally two eigenfunction expansions are derived.
\end{abstract}

1. Introduction. The most recent works which are pertinent to this article are those of Bryan [7], Tucker [22], and Vejvoda and Tvrdy [23], all of which discuss systems of the form

$$
x^{\prime}=A(t) x+f(t), \quad \int_{a}^{b} d F(t) x(t)=K,
$$

or slight generalizations of the same. In addition Brown [4], Brown and Krall [6], Brown, Green, and Krall [5], Green and Krall [11], and Krall [15], [16], [17] have considered the differential operator $L_{p} y=y^{\prime}+P y$ in the Hilbert space $\mathcal{L}_{n}^{2}(0,1)$ or the Banach space $\mathcal{L}_{n}^{p}(0,1), 1 \leq p<\infty$, where its domain is restricted by a boundary condition of the form

$$
\sum_{i=0}^{\infty} A_{i} y\left(t_{i}\right)=0,
$$

or

$$
\sum_{i=0}^{\infty} A_{i} y\left(t_{i}\right)+\int_{0}^{1} K(x) y(x) d x=0 .
$$

In these papers an adjoint has been either defined or derived, and in some of them eigenfunction expansions have been determined.

Further references can be found in the review articles by Whyburn [24] and Conti [8] as well as the article by Halanay and Moro [12].

The purpose of this article is to discuss in $\mathcal{L}_{n}^{p}(0,1)$ the boundary value problem

$$
l y=y^{\prime}+P y, \quad U(y)=\int_{0}^{1} d \nu(t) y(t)=0,
$$

where $P$ is an $n \times n$ matrix valued continuous function, $\nu$ is an $m \times n$ matrix

Received by the editors November 30, 1972.

AMS (MOS) subject classifications (1970). Primary 34B10.

(1) Sponsored in part by the U.S. Army, Contract No. DA-31-124-ARO-D-462. 
valued measure of bounded variation. The space $\mathcal{L}_{n}^{p}(0,1)$ consists of all $n$ dimensional vector valued functions defined on $[0,1]$ under the norm

$$
\|x\|_{p}=\left[\int_{0}^{1}\left\{\sum_{i=1}^{n}\left|x_{i}(t)\right|^{2}\right\}^{p / 2} d t\right]^{1 / p} .
$$

We remark without proof that $\mathcal{L}_{n}^{p}(0,1)$ has the usual properties associated with other complex $\mathcal{L}^{p}$ spaces. In particular, the dual of $\mathcal{L}_{n}^{p}(0,1), 1 \leq p<\infty$, is isometrically isomorphic to $\mathcal{L}_{n}^{q}(0,1), 1 / p+1 / q=1$. Hölder's inequality remains true: If $f \in \mathcal{L}_{n}^{p}(0,1)$ and $g \in \mathcal{L}_{n}^{q}(0,1)$, then

$$
\left|\int_{0}^{1} f^{*} g d t\right| \leq \int_{0}^{1}|f||g| d t \leq\|f\|_{p}\left\|_{g}\right\|_{q} .
$$

For a complete development of $\mathcal{L}^{p}$ functions with values in a Banach space see Lang [18] or Dinculeanu [9].

1.1. Definition. Let $D_{0 p}, 1 \leq p<\infty$, be the subspace of $\mathcal{L}_{n}^{p}(0,1)$ consisting of all elements satisfying

(a) $y$ is absolutely continuous on $[0,1]$ (hence, in fact, $y \in \mathcal{L}_{n}^{\infty}(0,1) \subset \mathcal{L}_{n}^{p}(0,1)$ );

(b) $l y=y^{\prime}+$ Py exists a.e. and is in $\mathcal{L}_{n}^{p}(0,1)$.

1.2. Definition. We define the differential operator $L_{0 p}$ by letting $L_{0 p} y=l y$ for all $y$ in $D_{0 p}$.

1.3. Definition. Let $U: D_{0 p} \rightarrow C^{m}$ be the operator $U(y)=\int_{0}^{1} d \nu(t) y(t)$. By $D_{p}$ we mean the subset of $D_{0 p}$ satisfying $U(y)=0$.

1.4. Definition. We define the differential operator $L_{p}$ by letting $L_{p} y=l y$ for all $y$ in $D_{p}$.

It is the operator $L_{p}$ which will be our primary concern.

At this point it is convenient to state some measure theoretic technicalities. As indicated earlier, the boundary functional $U$ is represented by an integral with respect to a matrix valued measure $\nu . \nu$ is understood to be a countably additive set function defined on the $\sigma$-ring of Lebesgue measurable sets in $[0,1]$ with range in the space of $m \times n$ matrices over the complex field $C$. Clearly $\nu$ can be represented by an $m \times n$ matrix $\left(\nu^{i j}\right)$ whose components are complex valued measures. By considering the components, many of the standard concepts of measure theory can be extended to the matrix valued case.

For example the total variation $\|\nu\|$ of $\nu$ may be defined by $\|\nu\|=\sup _{i j}\left\|\nu^{i j}\right\| . \nu$ is of bounded variation if $\|\nu\|<\infty$, which, following Tucker [22], we will assume to be true.

If $\nu$ and $\nu^{\prime}$ are two matrix valued measures, then $\nu$ is singular $\left(\nu \perp \nu^{\prime}\right)$ or absolutely continuous $\left(\nu \ll \nu^{\prime}\right)$ with respect to $\nu^{\prime}$ if and only if $\|\nu\| \perp\left\|\nu^{\prime}\right\|$ or $\|\nu\| \ll\left\|\nu^{\prime}\right\|$.

In addition a matrix valued measure $\nu$ will be singular $(\nu \perp \mu)$ or absolutely continuous $(\nu \ll \mu)$ with respect to Lebesgue measure if and only if $\|\nu\| \perp \mu$ or $\|\nu\| \ll \mu$.

If $y$ is an $n \times p$ matrix valued function and $\nu$ is an $m \times n$ matrix valued 
measure, then $y$ is $\nu$ measurable or $\nu$ integrable if every component of $y$ is measurable or integrable with respect to every component of $\nu$. For any measurable set $E$ in $[0,1]$, the integral of $y$ with respect to $\nu$ is given by

$$
\int_{E} d \nu(t) y(t)=\left(\sum_{k=1}^{n} \int_{E} y_{k j}(t) d \nu^{i k}(t)\right) .
$$

Such concepts sketched above lead to generalizations of the Lebesgue decomposition, Radon-Nikodym and Hopf-Carathéodory extension theorems which will be needed throughout the paper. Specifically, if $\nu$ is a matrix valued measure, then $\nu$ can be uniquely decomposed in the form $\nu=\nu_{c}+\nu_{s}$, where $\nu_{c}^{i j} \ll \mu$ and $\nu_{s}^{i j} \perp \mu$. ( $\nu_{s}$ can be further decomposed in the form $\nu_{s}=\nu_{s c}+\nu_{s a}$, where both $\nu_{s c}^{i j} \perp \mu$ and $\nu_{s a}^{i j} \perp \mu$, but $\nu_{s c}$ is continuous, while $\nu_{s a}$ is atomic (supported by points).)

There will also exist a unique $m \times n$ matrix valued function $d v_{c} / d \mu$ such that for each measurable set $E$

$$
\nu_{c}(E)=\int_{E} \frac{d v_{c}}{d \mu} d \mu
$$

We call $d v_{c} / d \mu$ the Radon-Nikodym derivative of $\nu_{c}$ with respect to $\mu$. It is an elementary exercise to show that $d v_{c} / d \mu=\left(d v_{c}^{i j} / d \mu\right)$ where $d v_{c}^{i j} / d \mu$ is the scalar Radon-Nikodym derivative of $\nu_{c}^{i j}$ with respect to $\mu$.

Dinculeanu [9] states the following generalization of the Hopf-Caratheodory extension theorem:

Let $A$ be an algebra of sets and $X$ be a Banach space. Then every countably additive set function of bounded variation $m: A \rightarrow X$ can be uniquely extended to a countably additive set function of bounded variation $\bar{m}: \delta \rightarrow X$, where $\delta$ is the $\sigma$ algebra generated by 4 .

$\bar{m}$ is the extension of $m$ from $\&$ to $\delta$. In our situation $X$ is the space of $m \times n$ matrices, and $\mathcal{A}$ is an algebra of intervals generating the Borel sets. $m$ then can be extended uniquely to a matrix valued Borel measure.

2. The density of $D_{p}$. It is well known that the adjoint of an operator on a normed vector space exists if and only if the domain of that operator is dense. This occurs in our case if and only if the kernel of the boundary functional $U$ is dense. When this happens, following Krall [14], we say that the boundary functional $U$ is acceptable.

Our purpose in this section is to characterize acceptable boundary functionals in terms of their representing measures. The results are stated in Theorems 2.6 and 2.8 .

Relying upon the Lebesgue decomposition and Radon-Nikodym theorems, we rewrite the boundary functional $U(y)=\int_{0}^{1} d \nu(t) y(t)$ as

$$
U(y)=\int_{0}^{1} d \nu_{s}(t) y(t)+\int_{0}^{1} \frac{d v_{c}}{d \mu}(t) y(t) d \mu,
$$


where $\nu_{s}$ and $\nu_{c}$ are unique, $\nu_{s} \perp \nu_{c}, \nu_{s} \perp \mu$ and $\nu_{c} \ll \mu$ on $[0,1]$, and $d \nu_{c} / d \mu$ is the Radon-Nikodym derivative of $\nu_{c}$ with respect to $\mu$. If $U$ is represented solely by a singular measure, we say $U$ is singular. Otherwise $U$ will be said to be mixed.

We state several principles of functional analysis as lemmas.

2.1. Lemma. A linear functional on a topological vector space is unbounded (discontinuous) if and only if its kernel is dense.

Proof. See Kelley and Namioka [13, p. 37].

2.2. Lemma (linear dependence principle). If $\phi, f_{1}, \ldots, f_{n}$ are linear functionals on a topological vector space, then $\phi=\sum_{i=1}^{n} \lambda_{i} f_{i}$ if and only if $\bigcap_{i=1}^{n} \operatorname{ker} f_{i} \subset \operatorname{ker} \phi$.

Proof. See Kelley and Namioka [13, p. 7].

2.3. Lemma (corollary to the Hahn-Banach theorem). If $K$ is a closed subspace of a normed (or locally convex) vector space, and if $p \notin K$, then there exists a continuous linear functional $\phi$ such that $K \subset \operatorname{ker} \phi$ and $\phi(p) \neq 0$.

Proof. See Royden [21, p. 190].

We also require the following two lemmas.

2.4. Lemma. Let $f_{1}, \ldots, f_{n}$ be a finite collection of linear functionals on a normed (or locally convex) vector space $V$. Then $\bigcap_{i=1}^{n}$ ker $f_{i}$ is dense in $V$ if and only if every linear combination $\sum_{i=1}^{n} \lambda_{i} f_{i} \neq 0$ is unbounded.

Proof. Assume $\bigcap_{i=1}^{n} \operatorname{ker} f_{i}$ is dense in $V$. Since

$$
\bigcap_{i=1}^{n} \operatorname{ker} f_{i} \subseteq \operatorname{ker} \sum_{i=1}^{n} \lambda_{i} f_{i} \subset V,
$$

ker $\sum_{i=1}^{n} \lambda_{i} f_{i}$ is dense in $V$. Hence, by Lemma $2.1, \sum_{i=1}^{n} \lambda_{i} f_{i}$ is unbounded.

Conversely, assume that every nontrivial linear combination $\sum_{i=1}^{n} \lambda_{i} f_{i}$ is unbounded, and that $\overline{\Pi_{i=1}^{n} \operatorname{ker} f_{i}}$ is strictly contained in $V$. Let $p \in V \backslash \overline{\bigcap_{i=1}^{n} \operatorname{ker} f_{i}}$. By Lemma 2.3 there exists a nonzero functional $\phi$ such that $\overline{\cap_{i=1}^{n} \operatorname{ker} f_{i}} \subset \operatorname{ker} \phi$, which is impossible by Lemma 2.2 .

2.5. Lemma. Let $U: V \rightarrow R^{m}$ be a densely defined operator on a normed (or locally convex) vector space $V$. Let $\pi_{i}, i=1, \ldots, m$, be the projection of $R^{m}$ onto its ith coordinate space. Then $\overline{\operatorname{ker} U}=V$ if and only if every nonzero functional $\sum_{i=1}^{m} \lambda_{i} \pi_{i}(U)$ is unbounded.

Proof. If $m=1$, the conclusion follows from Lemma 2.1. Otherwise, since ker $U=\bigcap_{i=1}^{m} \operatorname{ker} \pi_{i}(U)$, it follows from Lemma 2.4 .

2.6. Theorem. If $U$ is a singular boundary functional, then $U$ is acceptable.

Proof. We consider two cases.

(1) Let $n=1$. In this case since $D_{0 p}$ is dense if $1 \leq p<\infty$, according to Lemma 2.1 it is only necessary to verify that $U$ is unbounded. If we assume the 
contrary, then $U$ has a unique bounded extension $U$ on $\mathcal{L}_{n}^{p}(0,1)$. By the Riesz representation theorem, there exists an element $g$ in $\mathcal{L}_{n}^{q}(0,1), 1 / p+1 / q=1$, such that

$$
U(y)=\int_{0}^{1} g^{*}(t) y(t) d \mu(t)
$$

This implies that $d \nu_{s}=g^{*} d \mu$. Hence $\nu_{s} \ll \mu$, which is impossible.

(2) Let $n>1$, and let $J=\sum_{i=1}^{m} \lambda_{i} \pi_{i}(U)$ be a nontrivial linear combination. By Lemma 2.1 it is sufficient to show that $J$ is unbounded. Consider the subspace

$$
D_{p}^{j}=\left\{y: y \in D_{0 p} ; \pi_{k} y=0, k \neq j\right\} .
$$

That is, $D_{p}^{j}$ consists of all elements in $D_{0 p}$ with zero entries except possibly for $y_{j}$. Then

$$
\pi_{i}(U(y))=\int_{0}^{1} y_{j}(t) d v_{s}^{i j}(t)
$$

for all $y \in D_{p}^{j}$. By part (1), $\pi_{i}(U)$ is unbounded on $D_{p}^{j}$ and hence on $D_{0 p}$. Moreover, on an arbitrary set $D_{p}^{j}$

$$
J(y)=\sum_{i=1}^{m} \lambda_{i} \int_{0}^{1} y_{j}(t) d v_{s}^{i j}(t)=\int_{0}^{1} y_{j}(t) d \eta_{j}(t)
$$

when $\eta_{j}=\sum_{i=1}^{m} \lambda_{i} i_{s}^{i j}$. If $\eta_{j} \equiv 0$ for all $j$, then $J$ would be trivial on $D_{0 p}$, which contradicts our assumption. Thus, for some $j, \eta_{j}$ is a nonzero measure. It is obvious from the Lebesgue decomposition theorem that $\eta_{j} \perp \mu$. Hence, from part (1), $J$ is unbounded on $D_{p}^{j}$ and also on $D_{0 p}$.

Next we consider a mixed boundary functional.

2.7. Lemma. Let $B$ and $C$ be operators on a normed (or locally convex) vector space $V$ to $R^{m}$ such that $\overline{\operatorname{ker} B}=V, C$ is continuous, and $B+C$ is densely defined. Then $B+C$ is acceptable if and only if

$$
\sum_{i=1}^{m} \lambda_{i} \pi_{i}(B)=0 \text { implies } \sum_{i=1}^{m} \lambda_{i} \pi_{i}(C)=0 .
$$

Proof. Assume that $\sum_{i=1}^{m} \lambda_{i} \pi_{i}(B)=0$ implies $\sum_{i=1}^{m} \lambda_{i} \pi_{i}(C)=0$. By Lemma 2.5 it is sufficient to show that an arbitrary sum $\sum_{i=1}^{m} \lambda_{i} \pi_{i}(B+C) \neq 0$ is unbounded. Suppose the contrary. Since

$$
\sum_{i=1}^{m} \lambda_{i} \pi_{i}(B+C)=\sum_{i=1}^{m} \lambda_{i} \pi_{i}(B)+\sum_{i=1}^{m} \lambda_{i} \pi_{i}(C),
$$

we find that $\sum_{i=1}^{m} \lambda_{i} \pi_{i}(B)$ must be continuous. By Lemma 2.5 again $\sum_{i=1}^{m} \lambda_{i} \pi_{i}(B)$ $=0$. By assumption this implies $\sum_{i=1}^{m} \lambda_{i} \pi_{i}(C)=0$. Thus $\sum_{i=1}^{m} \lambda_{i} \pi_{i}(B+C)=0$, a contradiction.

The converse follows immediately from Lemma 2.5 . 
2.8. Theorem. The following statements are equivalent:

(1) $U$ is acceptable.

(2) If $\sum_{i=1}^{m} \lambda_{i} \pi_{i}\left(\int_{0}^{1} d v_{s}(t) y(t)\right)=0$ for all $y$ in $D_{0 p}$, then $\sum_{i=1}^{m} \lambda_{i} \pi_{i}\left(\int_{0}^{1} d v_{c}(t) y(t)\right)$ $=0$ for all $y$ in $D_{0 p}$.

Proof. Let $B y=\int_{0}^{1} d v_{s}(t) y(t)$ and $C y=\int_{0}^{1} d v_{c}(t) y(t)$ and apply Lemma 2.7.

2.9. Definition. Let $\nu$ be an $m \times n$ matrix valued measure of bounded variation. Let $T$ be a set in $[0,1]$. By the kernel $K_{\nu}^{T}$ of $\nu$ with respect to $T$ we mean $n_{t \in T} \operatorname{ker} \nu[0, t]$.

With the idea of the kernel of a measure it is possible to give an elegant characterization of an acceptable boundary functional.

2.10. Theorem. Let $T$ be everywhere dense in $[0,1]$. Then $U$ is acceptable if and only if $K_{v_{\xi}^{T}}^{T} \subset K_{p_{\epsilon}^{*}}^{T}$.

Proof. It is sufficient to show that the condition stated is equivalent to the second statement in Theorem 2.8. Let

$$
\sum_{i=1}^{m} \lambda_{i} \pi_{i}\left(\int_{0}^{1} d v_{s}(t) y(t)\right)=0
$$

imply

$$
\sum_{i=1}^{m} \lambda_{i} \pi_{i}\left(\int_{0}^{1} d \nu_{c}(t) y(t)\right)=0,
$$

and let $\phi$ be in $K_{v_{\xi}^{*}}^{T}$. Then $v_{s}^{*}[0, t] \phi=0$ on a dense set $T$. Let $\Psi=\sum_{i=1}^{k} \alpha_{i} \lambda\left(I_{i}\right)(t)$ be an arbitrary $C^{n}$ valued step function with ends in $T$. If $I_{i}=\left[a_{i}, b_{i}\right]$, then

$$
\begin{aligned}
\int_{0}^{1} \Psi^{*}(t) d v_{s}^{*}(t) \phi & =\sum_{i=1}^{k} \alpha_{i}^{*} \nu_{i}^{*}\left(I_{i}\right) \phi \\
& =\sum_{i=1}^{k} \alpha_{i}^{*} \nu_{i}^{*}\left[0, b_{i}\right] \phi-\sum_{i=1}^{k} \alpha_{i}^{*} \nu_{s}^{*}\left[0, a_{i}\right] \phi=0 .
\end{aligned}
$$

Since $T$ is dense in $[0,1]$, the step functions $\Psi$ are dense in $\mathcal{L}_{n}^{p}(0,1)$ and also in $D_{0 p}$. Thus

$$
\int_{0}^{1} y^{*}(t) d v_{s}^{*}(t) \phi=0
$$

for all $y$ in $D_{0 p}$. This can be rewritten in the form

$$
\sum_{i=1}^{m} \phi_{i} \pi_{i} \int_{0}^{1} d v_{s}(t) y(t)=0,
$$

where $\phi_{i}$ is the $i$ th component of $\phi$. By assumption this implies

$$
\sum_{i=1}^{m} \phi_{i} \pi_{i} \int_{0}^{1} d \nu_{c}(t) y(t)=0,
$$

which may also be rewritten as 


$$
\int_{0}^{1} y^{*}(t) d v_{c}^{*}(t) \phi=0
$$

Since this holds for all $y$ in $D_{0 p}$, it holds for all $y$ in $D_{0 p}$ with support in $[0, t]$. Moreover these elements are dense in $\mathcal{L}_{n}^{p}(0, t)$. These observations imply $v_{c}^{*}[0, t] \phi=0$ for all $t$ in $T$, or that $\phi \in K_{v_{c}^{*}}^{T}$.

Conversely, suppose that $K_{v_{s}^{*}}^{T} \subset K_{v_{c}^{*}}^{T}$. Then $v_{s}^{*}[0, t] \phi=0$ implies $v_{c}^{*}[0, t] \phi=0$. If

$$
\sum_{i=1}^{m} \lambda_{i} \pi_{i} \int_{0}^{1} d v_{s}(t) y(t)=0
$$

then

$$
\int_{0}^{1} y^{*}(t) d v_{s}^{*}(t) \lambda^{*}=0,
$$

where $\lambda=\left(\lambda_{1}, \ldots, \lambda_{m}\right)$. This implies (repeating the arguments above) that $u_{s}^{*}[0, t] \lambda^{*}=0$ for all $t$ in $T$. By assumption, this implies $v_{c}^{*}[0, t] \lambda^{*}=0$ for all $t$ in $T$, which in turn implies that

$$
\sum_{i=1}^{m} \lambda_{i} \pi_{i} \int_{0}^{1} d \nu_{c}(t) y(t)=0
$$

2.11. Corollary. If $K_{v^{*}}^{T}=0$, then the boundary functional $U$ is acceptable.

3. The adjoint of $L$. In this section we turn our attention to the construction of $L_{q}^{*}$, the adjoint of $L_{p}$, lying in $\mathcal{L}_{n}^{q}(0,1)$. For this purpose we will require a technical measure theoretic result depending upon the Hopf-Carathéodory extension theorem (see Royden [21, p. 257]).

3.1. Lemma. (1) If $T$ is everywhere dense and contains $\{1\}$, then the kernel of $\nu$ is invariant with respect to $T$. That is, if $T$ and $T$ are two everywhere dense sets containing $\{1\}$, then $K_{v}^{T}=K_{v}^{T}$.

(2) Let $T$ be everywhere dense and contain $\{1\}$, and let $\nu$ and $\nu^{\prime}$ be mutually singular. Then $K_{v-\nu}^{T} \subset K_{v}^{T} \cap K_{v}^{T}$.

Proof. (1) Let $A_{T^{\prime}}$ be the algebra generated by the interval $[0, t]$, where $t$ is in $T$. Suppose that $\phi$, in $C^{n}$, is in $K_{v}^{T}$ but not in $K_{v}^{T^{\prime}}$. Then $\nu \phi$ defines a nontrivial vector valued set function $\eta$ on $\mathcal{A}_{T^{\prime}}$ which may be uniquely extended to a measure $\bar{\eta}$ on the smallest $\sigma$-algebra $\mathcal{B}^{\prime}$ containing $\mathcal{A}_{T^{\circ}}$. Because $T$ is dense, $\mathcal{B}^{\prime}=\mathcal{B}$, the Borel sets in $[0,1]$. Because of uniqueness $\bar{\eta}$ is the measure $\nu \phi$ on $B$. But by the same reasoning, $\eta$ is also the unique extension of $\nu \phi$ restricted to $\mathcal{A}_{T}$. Since $T$ contains $1, \nu \phi$ is trivial. This contradiction shows that $K_{v}^{T} \subset K_{\nu}^{T^{\prime}}$. By interchanging $T$ and $T$, we find $K_{v}^{T}=K_{\nu}^{T}$.

(2) Let $\phi$ be in $K_{v-\nu}^{T}$. Then $\nu[0, t] \phi=\nu^{\prime}[0, t] \phi$. As in part (1), the set functions $\nu \phi$ and $\nu^{\prime} \phi$ agree on $\mathcal{A}_{T}$. Therefore their unique extensions to $B$ agree. But this contradicts the mutual singularity of $\nu$ and $\nu^{\prime}$ unless $\nu \phi$ and $\nu^{\prime} \phi$ are trivial on $A_{T}$, that is, unless $\phi$ is in $K_{\nu}^{T} \cap K_{\nu}^{T}$. 
In view of Lemma 3.1 we shall write $K$, to indicate the kernel of $\nu$ making no reference to the underlying at $T$, provided of course $T$ is dense and contains $\{1\}$.

3.2. Definition. In $\mathcal{L}_{n}^{q}(0,1), 1 \leq q \leq \infty$, let $D_{0_{q}}^{+}$be the set of absolutely continuous functions $z$ vanishing at $t=0, t=1$, such that $l_{0}^{+} z=-z^{\prime}+P^{*} z$ is in $\mathcal{L}_{n}^{q}(0,1)$.

3.3. Definition. We define the differential operator $L_{0 q}^{+}$by letting $L_{0 q}^{+} z=l_{0}^{+} z$ for all $z$ in $D_{0 q}^{+}$.

3.4. Definition. In $\mathcal{L}_{n}^{q}(0,1), 1 \leq q \leq \infty$, let $D_{q}^{+}$be the set of all elements $z$ for which there exists a vector $\phi(z)$ in the quotient space $C^{m} / K_{v_{s}}$ such that

(1) $z(t)=\varepsilon(t)-\nu_{s}^{*}[0, t] \phi(z)$ where $\varepsilon(t)$ is absolutely continuous.

(2) $l^{+} z=-z^{\prime}+P^{*} z+\left(d v_{c} / d \mu\right)^{*} \phi(z)$ exists a.e. in $\mathcal{L}_{n}^{q}(0,1)$.

(3) $z(0+)=-v_{s}^{*}[0] \phi(z), z(1-)=v_{s}^{*}[1] \phi(z)$.

3.5. Definition. We define the differential-boundary operator $L_{q}^{+}$by letting $L_{q}^{+} z=l^{+} z$ for all $z$ in $D_{q}^{+}$.

The principal result to follow in this section is that $L_{p}$ and $L_{q}^{+}, 1 \leq p<\infty$, $1 / p+1 / q=1$, are dual operators.

3.6. Lemma. The mapping $\eta: D_{q}^{+} \rightarrow C^{m} / K_{v^{*}}$ defined by $\eta(z)=\phi(z)$ is a welldefined linear operator. $\eta(z)=0$ if and only if $z$ is in $D_{0 q}^{+}$. The expression $l^{+} z$ is well defined if and only if the domain of $L_{p}, D_{p}$, is dense in $\mathcal{L}_{n}^{p}(0,1)$.

Proof. Suppose that $\eta$ maps $z$ onto two elements of $C^{m} / K_{v^{*}}, \phi_{1}(z)$ and $\phi_{2}(z)$. Then by condition (1) of Definition 3.4, $z(t)+v_{s}^{*}[0, t] \phi_{1}(z)$ and $z(t)+v_{s}^{*}[0, t] \phi_{2}(t)$ are absolutely continuous. Subtracting, we find $v_{s}^{*}[0, t]\left(\phi_{1}(z)-\phi_{2}(z)\right)$ is absolutely continuous, which is impossible unless it is the zero measure. Therefore $\phi_{1}(z)-\phi_{2}(z)$ is in $K_{v_{\xi}^{*}}$. That $\eta$ is linear and onto is trivial.

Secondly, if $z$ is in $D_{0 p}$ and $z(t)+\nu_{s}^{*}[0, t] \phi(z)$ is absolutely continuous, then $v_{s}^{*}[0, t] \phi(z)$ is the zero measure, and $\phi(z)$ is zero in $C^{m} / K_{v_{j}^{*}}$. Conversely, if $\phi(z)=0$ in $C^{m} / K_{v_{s}^{*}}$, then $v_{s}^{*}[0, t] \phi=0, z$ is absolutely continuous and vanishes at 0 and 1 by parts (1) and (3) of Definition 3.4.

Finally that $l^{+}$is well defined if and only if $D_{p}$ is dense follows from Theorem 2.10.

3.7. Theorem. The differential-boundary operator $L_{q}^{+}$is closed, $1<q \leq \infty$.

Proof. Recall that $L_{q}^{+}$is closed if whenever $\lim _{k \rightarrow \infty} z_{k}=z\left(z_{k} \in D_{k}^{+}\right)$and $\lim _{k \rightarrow \infty} L_{q}^{+} z_{k}=y$, then $z$ is in $D_{k}^{+}$and $L_{q}^{+} z=y$.

Now

Since

$$
\begin{aligned}
z_{k}(s)-z_{l}(s)= & \int_{0}^{s}\left(z_{k}^{\prime}-z_{l}^{\prime}\right) d \xi-v_{s}^{*}[0, s] \phi\left(z_{k}-z_{l}\right) \\
= & -\int_{0}^{s} L_{q}^{+}\left(z_{k}-z_{l}\right) d \xi+\int_{0}^{s} P^{*}\left(z_{k}-z_{l}\right) d \xi \\
& +\left[v_{c}^{*}[0, s]-v_{s}^{*}[0, s]\right] \phi\left(z_{k}-z_{l}\right) .
\end{aligned}
$$

$$
\left|\int_{0}^{s} L_{q}^{+}\left(z_{k}-z_{l}\right) d \xi\right| \leq\left\|L_{q}^{+}\left(z_{k}-z_{l}\right)\right\|_{q}
$$


and

$$
\left|\int_{0}^{s} P^{*}\left(z_{k}-z_{l}\right) d \xi\right| \leq\left\|P^{*}\right\|_{p}\left\|z_{k}-z_{l}\right\|_{q},
$$

we find that $z_{k}(s)+\left[z_{s}^{*}[0, s]-v_{c}^{*}[0, s]\right] \phi\left(z_{k}\right)$ converges uniformly on $[0,1]$. Since $z_{k}(1-)=v_{s}^{*}[1] \phi\left(z_{k}\right)$ and $z_{k}(0+)=-v_{s}^{*}[0] \phi\left(z_{k}\right),\left[\nu_{s}^{*}[0,1]-v_{c}^{*}[0,1]\right] \phi\left(z_{k}\right)$ and $v_{s}^{*}[0]_{\phi}\left(z_{k}\right)$ converge. Further if we take $q$-norms on the inequalities above, we find

$$
\left\|\int_{0}^{s} L_{q}^{+}\left(z_{k}-z_{l}\right) d \xi\right\|_{q} \leq\left\|L_{q}^{+}\left(z_{k}-z_{l}\right)\right\|_{q}
$$

and

$$
\left\|\int_{0}^{s} P^{*}\left(z_{k}-z_{l}\right) d \xi\right\|_{q} \leq\left\|P^{*}\right\|_{p}\left\|z_{k}-z_{l}\right\| .
$$

Thus the original equation yields

$\left\|\left[\nu_{c}^{*}[0, s]-z_{s}^{*}[0, s]\right]_{\phi}\left(z_{k}-z_{l}\right)\right\|_{q} \leq\left\|z_{k}-z_{l}\right\|_{q}+\left\|P^{*}\right\|_{p}\left\|z_{k}-z_{l}\right\|_{q}+\left\|L_{q}^{+}\left(z_{k}-z_{l}\right)\right\|_{q}$,

which shows that the functions

$$
F_{k}(s)=\left[\nu_{c}^{*}[0, s]-v_{s}^{*}[0, s]\right] \phi\left(z_{k}\right)
$$

converge in $\mathcal{L}_{n}^{q}(0,1)$ and thus also in measure. Thus there exists a subsequence $F_{m}(s)$ which converges on a set $T$ of unit measure. Clearly $T$ is everywhere dense and contains $\{1\}$. Thus, by Lemma 3.1, $K_{v_{i}^{*} v_{c}^{*}}^{T} \subset K_{v_{\varepsilon}^{*}}^{T}$ and $\left\{\phi\left(z_{m}\right)\right\}$ is a Cauchy sequence in $C^{m} / K_{v^{*}}$. In other words, we have shown that a subsequence $\left\{\phi\left(z_{m}\right)\right\}$ converges to an element $\phi$ in $C^{m} / K_{v_{s}^{*}}$. Since

$$
z_{m}(s)=-\int_{0}^{s} L_{q}^{+} z_{m} d \xi+\int_{0}^{s} P^{*} z_{m} d \xi+\left[\nu_{c}^{*}[0, s]-v_{s}^{*}[0, s] \phi\left(z_{m}\right),\right.
$$

and $\phi\left(z_{m}\right)$ converges, we may take limits to find

$$
z(s)=-\int_{0}^{s} y d \xi+\int_{0}^{s} P^{*} z d \xi+\left[\nu_{c}^{*}[0, s]-v_{s}^{*}[0, s]\right] \phi,
$$

or

$$
z(s)+v_{s}^{*}[0, s] \phi=-\int_{0}^{s} y d \xi+\int_{0}^{s} P^{*} z d \xi+v_{c}^{*}[0, s] \phi .
$$

Since the right side is absolutely continuous, $z$ satisfies the first requirement to be in $D_{q}^{+}$. Differentiating, we find

$$
y=-z^{\prime}+P^{*} z+\left(d v_{c} / d \mu\right)^{*} \phi \text { a.e., }
$$

the second condition. The third condition follows trivially.

3.8. Lemma. $\left(L_{0_{q}}^{+}\right)^{*}=L_{0 p}, 1 \leq q<\infty$. 
Proof. This is well known. For example, see Goldberg [10, Chapter VI] for a proof with $n=1$. His proof is easily modified to fit the present situation.

3.9. Theorem. $\left(L_{q}^{+}\right)^{*}=L_{p}, 1<p \leq \infty$.

Proof. If $1<p \leq \infty$, then $1 \leq q<\infty$. Consequently $D_{0 q}^{+}$is dense in $\mathcal{L}_{n}^{q}(0,1)$. Since $D_{D_{q}}^{+} \subset D_{q}^{+},\left(L_{q}^{+}\right)^{*}$ is well defined.

First we show that $L_{p} \subset\left(L_{q}^{+}\right)^{*}$. If $y \in D_{p}$ and $z \in D_{q}^{+}$, then

$$
\begin{aligned}
\left(L_{p} y, z\right)-\left(y, L_{q}^{+} z\right)= & \int_{0}^{1}\left[z^{*}(s)\left(L_{p} y(s)\right)-\left(L_{q}^{+} z(s)\right)^{*} y(s)\right] d s \\
= & \int_{0}^{1}\left[z(s)^{*} y^{\prime}(s)+z^{\prime}(s)^{*} y(s)+\left(\frac{d v_{c}^{*}}{d \mu}(s) \phi(z)\right)^{*} y(s)\right] d s \\
= & \int_{0}^{1}\left[z(s)+v_{s}^{*}[0, s] \phi(z)\right]^{*} y^{\prime}(s) d s-\int_{0}^{1}\left[z_{s}^{*}[0, s] \phi(z)\right]^{*} y^{\prime}(s) d s \\
& +\int_{0}^{1}\left[z(s)+v_{s}^{*}[0, s] \phi(z)\right]^{* \prime} y(s) d s-\int_{0}^{1}\left[v_{s}^{*}[0, s] \phi(z)\right]^{* \prime} y(s) d s \\
& +\int_{0}^{1}\left[\frac{d v_{c}^{*}}{d \mu}(s) \phi(z)\right] y(s) d s .
\end{aligned}
$$

Since $y(s)$ and $z(s)+v_{s}^{*}[0, s] \phi(z)=\varepsilon(s)$ are absolutely continuous, the sum of the first and third integrals is

$$
\left.\varepsilon(s) y(s)\right|_{s=0+} ^{s=1-}=\phi(z)^{*} v_{s}[0,1] y(1) .
$$

The second integral may be written as

$$
-\phi(z)^{*} \int_{0}^{1} v_{s}[0, s] y^{\prime}(s) d s .
$$

Its value is

$$
\phi(z)^{*} v_{s}[0] y(0)-\phi(z)^{*}{ }_{v}[0,1] y(1)+\phi(z)^{*} \int_{0}^{1} d v_{s}(s) y(s) .
$$

The fourth integral is $\mathbf{0}$. Finally the last integral is

$$
\phi(z)^{*} \int_{0}^{1} \frac{d v_{c}}{d \mu}(s) y(s) d s
$$

Adding, we find

$$
\begin{aligned}
\left(L_{p} y, z\right)-\left(y, L_{q}^{+} z\right) & =\phi(z)^{*}\left[\int_{0}^{1} d v_{s}(s) y(s)+\int_{0}^{1} \frac{d v_{c}}{d \mu}(s) y(s) d s\right] \\
& =\phi(z)^{*} \int_{0}^{1} d v(s) y(s)=0 .
\end{aligned}
$$

To show the reverse inclusion, let $z \in D_{0 q}^{+}$; then since $D_{0_{q}}^{+} \subset D_{q}^{+}$and $\phi(z)=0$ for such z, $L_{0_{q}}^{+} \subset L_{q}^{+}$. This implies $\left(L_{q}^{+}\right)^{*} \subset\left(L_{0_{q}^{+}}^{+}\right)^{*}=L_{0 p}$. This implies that the domain of $\left(L_{q}^{+}\right)^{*}$ is in $D_{0 p}$. For arbitrary $z \in D_{q}^{+}$and $y \in D_{0 p}$ the calculation in 
the first part of the proof shows $\phi(z)^{*} \int_{0}^{1} d \nu(s) y(s)=0$. Since $\phi$ maps $D_{q}^{+}$onto $\left.C^{m},{ }^{2}\right) \int_{0}^{1} d v(s) y(s)=0$. Thus the domain of $\left(L_{q}^{+}\right)^{*}$ satisfies the boundary condition and is in $D_{p}$. Therefore $\left(L_{q}^{+}\right)^{*} \subset L_{p}$, and the two are equal.

\subsection{Theorem. If $1 \leq p<\infty, L_{p}^{*}=L_{q}^{+}$.}

Proof. We consider two cases.

Let $p>1$. Then $D_{q}^{+}$, the domain of $L_{q}^{+}$is dense, and $\mathcal{L}_{n}^{q}(0,1)$ is reflexive. Further $L_{q}^{+}$is closed. It follows then that $\left(L_{q}^{+}\right)^{* *}=L_{q}^{+}$. Since, by Theorem 3.9; $\left(L_{q}^{+}\right)^{*}=L_{p}, L_{p}^{*}=\left(L_{q}^{+}\right)^{* *}=L_{q}$.

Let $p=1$; since $\mathcal{L}_{n}^{\sigma}(0,1) \subset \mathcal{L}_{n}^{1}(0,1)$ for $\sigma>1$, it follows that $L_{\sigma} \subset L_{1}$ and $L_{1}^{*} \subset L_{\sigma}^{*}=L_{\tau}^{+}(1 / \sigma+1 / \tau=1)$. Thus $L_{1}^{*} z=l^{+} z$ on some domain $S \subseteq D_{\tau}^{+}$ $\cap \mathcal{L}_{n}^{\infty}(0,1)$. On the other hand, a simple calculation similar to that of Theorem 3.9 shows that $L_{\infty}^{+} \subset L_{1}^{*}$, i.e., that $D_{\infty}^{+} \subset S$. Since $D_{\tau}^{+} \cap \mathcal{L}_{n}^{\infty}(0,1)=D_{\infty}^{+}, S$ $=D_{\infty}^{+}$, and $L_{1}^{*}=L_{\infty}^{+}$.

4. Spectral analysis-expansions. Brown [3] has shown that, when $\nu_{s}$ is purely atomic, the operators $L_{p}$ and $L_{q}^{+}$are Fredholm operators (that is, they have closed ranges, finite dimensional kernels and deficiencies, and mutually orthogonal ranges and kernels), and that when $m=n$ there is only point spectrum. Furthermore if $\nu_{c}=0$, it is easy to show that the eigenvalues are isolated (see [11]). Since the proofs behind these results depend in no way upon the nature of the representing measures, they remain valid in our setting. In other words $L_{p}$ and $L_{q}^{+}$are Fredholm. When $m=n$, the spectrum of $L_{p}$ (and $L_{q}^{+}$) consists only of isolated eigenvalues. If $\lambda$ is not one of them, then $L_{p}-\lambda I$ is invertible, the resolvent being generated by an integral operator with a Green's function as its kernel. It is these facts we now wish to investigate in more detail. We emphasize that throughout the remainder of the paper $m=n$.

In addition we need to assume that $\nu_{s}$ is atomic at $t=0$ and $t=1$, and that $v_{s}[0]=A_{0}, v_{s}[1]=A_{1}$ are nonsingular matrices.

Eigenvalues. If we let $Y(t)$ be a fundamental matrix for the differential equation $y^{\prime}+P y=0$, then an elementary calculation shows that $y(t)=Y(t) e^{\lambda t}$ satisfies $y^{\prime}+P y=\lambda y$. Eigenvalues will then be the roots of

$$
\operatorname{det}\left(U\left(Y(t) e^{\lambda t}\right)\right)=\operatorname{det}\left(\int_{0}^{1} d \nu(t) Y(t) e^{\lambda t}\right)=0 .
$$

If $\lambda$ does not satisfy the equation above, then

$$
y(t)=\int_{0}^{1} G_{\lambda}(t, s) f(s) d s
$$

where

$$
G_{\lambda}(t, s)=Y(t) e^{\lambda t} U\left(Y(t) e^{\lambda t}\right)^{-1} M(t, s) Y(s)^{-1} e^{-\lambda s},
$$

$\left(^{2}\right)$ In the sense that $U_{z \in D_{i}^{+}} \phi(z)^{*}=C^{m}$. 


$$
\begin{array}{rlrl}
M(t, s) & =\int_{0}^{s} d \nu(\xi) Y(\xi) e^{\lambda \xi}, & 0 \leq s \leq t \leq 1, \\
& =-\int_{s}^{1} d \nu(\xi) Y(\xi) e^{\lambda \xi}, \quad 0 \leq t \leq s \leq 1,
\end{array}
$$

provides a solution to $y^{\prime}+P y-\lambda y=f$ which also satisfies $U(y)=0$. Since $G_{\lambda}$ is bounded, the integral operator is itself a bounded operator on $\mathcal{L}_{n}^{p}(0,1)$.

4.1.Theorem. The spectrum of $L_{p}$ consists only of an infinite number of eigenvalues $\lambda$ which can accumulate only at $\infty$. The eigenvalues are the zeros of

$$
\operatorname{det} U\left(Y(t) e^{\lambda t}\right)=\operatorname{det}\left(\int_{0}^{1} d \nu(t) Y(t) e^{\lambda t}\right) .
$$

The eigenvalues all lie in a vertical strip $|\operatorname{Re} \lambda|<h$. Within that strip, the number of eigenvalues lying within the rectangle $|\operatorname{Re} \lambda|<h, R<\operatorname{Im} \lambda<k+1$, is bounded by a number $M$ which is independent of $k$. Finally, for any $\delta>0$, there exists a number $m(\delta)>0$ such that

$$
\left|\operatorname{det}\left(U\left(Y(t) e^{\lambda t}\right)\right)\right|>m(\delta)
$$

for all $\lambda$ within the strip $|\operatorname{Re} \lambda|<h$ lying outside circles of radius $\delta$ centered at the eigenvalues.

Proof. Clearly if det $\left(U\left(Y(t) e^{\lambda t}\right)\right)=0$, then $\lambda$ is an eigenvalue with at least one eigenfunction. Since det $\left(U\left(Y(t) e^{\lambda t}\right)\right)$ is holomorphic in $\lambda$, the eigenvalues can accumulate only at $\infty$. Further if $\lambda$ is not a zero, then $\lambda$ is in the resolvent set.

As $\operatorname{Re} \lambda \rightarrow+\infty$, we note, due to the atomic nature of $\nu(1)$, that

$$
U\left(Y(t) e^{\lambda t}\right)=A_{1} Y(1) e^{\lambda}(1+o(1)) .
$$

This is ultimately never singular since $A_{1}$ is nonsingular. Similarly, as $\operatorname{Re} \lambda$ $\rightarrow-\infty$,

$$
U\left(Y(t) e^{\lambda t}\right)=A_{0} Y(0) e^{0}(1+o(1))
$$

which is also nonsingular. Thus the eigenvalues lie within some vertical strip $|\operatorname{Re} \lambda|<h$.

Within that strip it is possible (see Green and Krall [11]) to transform $\operatorname{det}\left(U\left(Y(t) e^{\lambda t}\right)\right)$ into an entire function of nonintegral order which, following Boas [2, p. 24], has an infinite number of zeros.

Finally the estimates concerning the number of zeros within a rectangular region and the minimum modulus of $\left|\operatorname{det}\left(U\left(Y(t) e^{\lambda t}\right)\right)\right|$ are direct quotes from Levin [19, pp. 268-269]. Only cursory inspection is necessary to see that the function is almost periodic.

The spectrum of $L_{q}^{+}$also consists only of eigenvalues $\bar{\lambda}$, where $\lambda$ is an eigenvalue of $L_{p}$. Bryan [7] has characterized the eigenfunctions associated with them: 
4.2. Theorem. The spectrum of $L_{q}^{+}$consists only of eigenvalues $\bar{\lambda}$,where $\lambda$ is an eigenvalue of $L_{p}$. The corresponding eigenfunctions are given by

$$
z(t)=-Y^{*}(t)^{-1} e^{-\bar{\lambda} t} \int_{0}^{t} e^{\bar{\lambda} s} Y^{*}(s) d \nu^{*}(s) \phi(z)
$$

where $\phi(z) \in \operatorname{ker} U\left(Y(t) e^{\lambda t}\right)^{*}$.

Proof. An elementary calculation shows that

$$
-z^{\prime}+P^{*} z+\left(d v_{c} / d \mu\right)^{*} \phi=\bar{\lambda} z \text { a.e. }
$$

That $z(t)+v_{s}^{*}[0, t] \phi(z)$ is absolutely continuous also follows from the formula, as does the equation $z(0+)=-v_{s}^{*}[0] \phi(z)$. Finally, we calculate

$$
\begin{aligned}
z(1-) & =-Y^{*}(1)^{-1} e^{-\bar{\lambda}} \int_{0}^{1-} e^{-\bar{\lambda} s} Y^{*}(s) d \nu^{*}(s) \phi(z) \\
& =-Y^{*}(1)^{-1} e^{-\bar{\lambda}}\left[U\left(Y(t) e^{\lambda t}\right)\right]^{*} \phi(z)+\nu_{s}^{*}[1] \phi(z) \\
& =\nu_{s}^{*}[1] \phi(z) .
\end{aligned}
$$

Eigenmanifolds. If $\lambda$ is a multiple eigenvalue, then not only do we find eigenfunctions, but also other related functions which generate what is usually called an eigenmanifold. These are most naturally generated by examining the poles of the Green's function. They enter also as terms in an eigenfunction expansion, first for the Green's function, second for functions in $D_{p}$ and $D_{q}^{+}$.

Since the results are almost identical with those found in [17] we quote the results without proof.

4.3. Theorem. Let $\lambda_{0}$ be in the resolvent set of $L_{p}$, and let $\lambda_{n}$ be an $m$-fold zero of $\operatorname{det}\left(U\left(Y(t) e^{\lambda t}\right)\right)$. Near $\lambda_{n}$ let

$$
\begin{aligned}
Y(t) e^{\lambda t} & =\sum_{j=0}^{\infty} u_{j}\left(\lambda-\lambda_{n}\right)^{j} \\
M(t, s) Y(s)^{-1} e^{-\lambda s} & =\sum_{k=0}^{\infty} v_{k}^{*}\left(\lambda-\lambda_{n}\right)^{k} \\
U\left(Y(t) e^{\lambda t}\right)^{-1} & =\sum_{l=0}^{\infty} U_{l}\left(\lambda-\lambda_{n}\right)^{l-m}
\end{aligned}
$$

Then the residue of $G_{\lambda}(t, s) /\left(\lambda-\lambda_{0}\right)$ at $\lambda_{n}$ is

$$
-\sum_{j=0}^{m-1} \sum_{k=0}^{m-1-j} \sum_{l=0}^{m-1-j-k} u_{j} U_{l} v_{k}^{*}\left(\lambda_{0}-\lambda_{n}\right)^{m-j-k-l}
$$

The elements $u_{j}, j=0, \ldots, m-1$, are in $D_{p}$; the elements $v_{k}, k=0, \ldots, m-1$, are in $D_{q}^{+}$and are functions of $s$ alone. They satisfy 


$$
\begin{array}{rlrl}
\left(L_{p}-\lambda_{n} I\right) u_{0} & =0, & \\
\left(L_{p}-\lambda_{n} I\right) u_{j} & =u_{j-1}, & & j=1, \ldots, m-1 . \\
\left(L_{q}^{+}-\bar{\lambda}_{n} I\right) v_{0} & =0 & & \text { a.e. } \\
\left(L_{q}^{+}-\bar{\lambda}_{n} I\right) v_{k} & =v_{k-1} & & \text { a.e. }, k=1, \ldots, m-1 .
\end{array}
$$

$\left\{u_{j}\right\}_{j=1}^{m-1}$ and $\left\{v_{k}\right\}_{k=0}^{m-1}$ form linearly independent sets. For different eigenvalues, the linear subspaces spanned by the sets $\left\{u_{j}\right\}$ and $\left\{v_{k}\right\}$ are mutually orthogonal. That is, $\int_{0}^{1} v_{k} u_{j} d \mu=0$.

Expansion of the Green's function.

4.4. Theorem. Let the eigenvalues of $L_{p}$ be denoted by $\left\{\lambda_{n}\right\}_{n=1}^{\infty}$, where their orders $m$ and elements $u_{j}, U_{l}, v_{k}$ are dependent upon $n$. If $\lambda$ is in the resolvent set for $L_{p}$, then

$$
G_{\lambda_{0}}(t, s)=\sum_{n=0}^{\infty} \sum_{j=0}^{m-1} \sum_{k=0}^{m-1-j} \sum_{l=0}^{m-1-j-k} u_{j}(t) U_{l} v_{k}^{*}(s) /\left(\lambda_{0}-\lambda_{n}\right)^{m-j-k-l} .
$$

Proof. Estimates similar to those found in [17] show that as $|\operatorname{Re} \lambda| \rightarrow \infty$, the Green's function approaches 0 . If $\phi\left[G_{\lambda}(t, s) /\left(\lambda-\lambda_{0}\right)\right] d \lambda$ is computed around a contour which approaches $\infty$ while uniformly avoiding the eigenvalues (as is possible according to Theorem 4.1), then Cauchy's residue theorem yields the result above (see [15] or [17] for details).

Eigenfunction expansions. By using the expansion of the Green's function two eigenfunction expansions can be derived: one for elements in $D_{p}$, one for elements in $D_{q}^{+}$. In addition, Parseval's equality for one element in $D_{p}$, the other in $\mathcal{L}_{n}^{q}(0,1)$, or for one element in $\mathcal{L}_{n}^{p}(0,1)$, the other in $D_{q}^{+}$, then follows immediately.

4.5. Theorem. Let $y$ be in $D_{p}$. Then

$$
y(t)=-\sum_{n=1}^{\infty} \sum_{j=0}^{m-1} \sum_{k=0}^{m-1-j} u_{j}(t) U_{m-1-j-k} \int_{0}^{1} v_{k}^{*}(s) y(s) d s .
$$

4.6. Theorem. Let $z$ be in $D_{q}^{+}$. Then

$$
z(s)=-\sum_{n=1}^{\infty} \sum_{j=0}^{m-1} \sum_{k=0}^{m-1-j} v_{k}(s) U_{m-1-j-k}^{*} \int_{0}^{1} u_{j}^{*}(t) z(t) d t .
$$

The proofs of these theorems are similar to those found in [17]. Likewise the following also holds:

4.7. Theorem. If $y$ is in $D_{p}$ and $z$ is in $\mathcal{L}_{n}^{q}(0,1)$, or if $y$ is in $\mathcal{L}_{n}^{p}(0,1)$ and $z$ is in $D_{q}^{+}$, then

$$
\int_{0}^{1} z^{*}(u) y(u) d u=-\sum_{n=1}^{\infty} \sum_{j=0}^{m-1} \sum_{k=0}^{m-1-j} \int_{0}^{1} z^{*}(t) u_{j}(t) d t U_{m-1-j-k} \int_{0}^{1} v_{k}^{*}(s) y(s) d s .
$$


5. Selfadjoint systems. Under special circumstances it is possible for the problem

$$
L_{p} y=y^{\prime}+P y, \quad \int_{0}^{1} d \nu(t) y(t)=0,
$$

to possess a type of selfadjointness when $p=2$. The idea, due originally to Bliss [1] and substantially extended by Reid [20], is that while the system and its adjoint may not be the same, it might be possible for there to exist a nonsingular transformation $\square$ which transforms one system into the other. Under $\square$ eigenfunctions would be transformed into each other. Since the eigenvalues will be the same however, they will all have to be real. In order for this to happen, we introduce the continuous matrix $R$, mainly with the idea of rotating the eigenvalues discussed earlier by $90^{\circ}$ so that they are now bounded vertically and can possibly lie on the real axis.

In order to apply the concept, we rephrase the boundary value problem and its adjoint problem in a new matrix notation. We find, upon letting $u(t)$ $=\int_{0}^{t} d v_{c}(s) y(s)$, that the problem

$$
\begin{aligned}
y^{\prime}+P y & =\lambda R y, \\
\int_{0}^{1} d v(t) y(t) & =\int_{0}^{1}\left(\frac{d v_{c}}{d \mu}\right)(t) y(t) d t+\int_{0}^{1} d v_{s}(t) y(t)=0,
\end{aligned}
$$

is equivalent to the problem

$$
Y^{\prime}+\mathscr{P} Y=\lambda \propto R Y, \quad \int_{0}^{1} d V(t) Y(t)=0
$$

where

$$
Y=\left(\begin{array}{l}
y \\
u
\end{array}\right), \quad \mathscr{P}=\left(\begin{array}{cc}
p & 0 \\
-\nu_{c}^{\prime} & 0
\end{array}\right), \quad \mathcal{R}=\left(\begin{array}{ll}
R & 0 \\
0 & 0
\end{array}\right), \quad \text { and } \quad V=\left(\begin{array}{cc}
\nu_{s} & a_{1} \\
0 & a_{0}
\end{array}\right),
$$

where $a_{0}$ and $a_{1}$ are matrix valued atomic measures, $a_{0}[0]=I, a_{1}[1]=I$, but all zero at all other points.

Similarly, the adjoint problem

$$
\begin{aligned}
& -z^{\prime}+P^{*} z+\left(\frac{d v_{c}}{d \mu}\right)^{*} \phi(z)=\lambda R^{*} z \text { a.e., } \\
& z(t)+v_{s}^{*}[0, t] \phi(z) \text { is absolutely continuous, } \\
& z(0+)=-v_{s}^{*}[0]_{\phi}(z), z(1-)=v_{s}^{*}[1] \phi(z),
\end{aligned}
$$

is equivalent to the problem

$$
\begin{aligned}
& -Z^{\prime}+\mathscr{P}^{*} Z=\lambda \propto R^{*} Z \text { a.e., } \\
& Z(t)+V^{*}[0, t] \Phi(z) \text { is absolutely continuous, } \\
& Z(0+)=-V^{*}[0] \Phi(Z), Z(1-)=V^{*}[1] \Phi(Z),
\end{aligned}
$$


where $Z=\left(\begin{array}{l}z \\ v\end{array}\right), v=-\phi(z)$ and $\Phi(Z)=\left(\begin{array}{l}\phi z) \\ \phi(z)\end{array}\right)$. Note that now the measure $V$ is singular. It contains no absolutely continuous part.

Symmetric systems. Let us now investigate the possibility of the existence of a transformation $\supset$ between these problems.

\subsection{Definition. The system}

$$
Y^{\prime}+\mathcal{P} Y=\lambda \propto R Y, \quad \int_{0}^{1} d V(s) Y(s)=0
$$

is symmetric if there exists a nonsingular matrix transformation of bounded variation $\Xi$ such that under $Z=\square Y$ the system is equivalent to its adjoint system

$$
\begin{aligned}
& -Z^{\prime}+\mathscr{P}^{*} Z=\lambda \sigma R Z, \\
& Z(t)+\mathcal{V}^{*}[0, t] \Phi(z) \quad \text { is absolutely continuous, } \\
& Z(0+)=-\mathcal{V}^{*}[0] \Phi(z), Z(1-)=\mathcal{V}^{*}[1] \Phi(z),
\end{aligned}
$$

for all eigenvalues $\lambda$.

Suppose that such a transformation $\square$ exists. If $\mathcal{Y}$ is a fundamental matrix for $Y^{\prime}+\mathscr{P} Y=\lambda \mathcal{R} Y$, then $\mathcal{Z}=\square Y$ satisfies the adjoint differential equation. Substitution yields

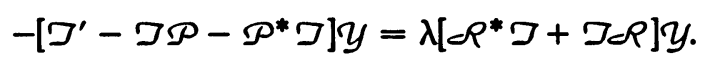

If $\beth$ is to transform eigenfunctions for all eigenvalues, then it is convenient to require $\beth^{\prime}=\supset \mathscr{P}+\mathscr{P}^{*} \supset, 0=\mathcal{R}^{*} \beth+\beth \subset \mathcal{~}$.

Since $\square Y+\mathcal{V}^{*}[0, t] \Phi(\square Y)$ is to be absolutely continuous, after substituting $Y(t)=Y(t) Y_{0}$, where $Y_{0}$ is constant, we are led to expect the singular part of $d \square$ should satisfy

$$
d \beth_{s}(t) \mathcal{Y}(t) Y_{0}=-d \mathcal{V}^{*}(t) \Phi(\supset Y) .
$$

If $Y$ is chosen properly, we can require $Y_{0}=\Phi(\supset Y)$ and

$$
d \beth_{s}(t)=-d V^{*}(t) y(t)^{-1} \text {. }
$$

Further this differential $d \beth_{s}$ should be the same for all eigenvalues $\lambda$.

Finally, since the $Z$ boundary conditions at 0 and 1 imply $Z(0-)=Z(1+)$ $=0$, we should require $\supset(0-)=\supset(1+)=0$. This can be further checked if we use Green's formula:

$$
\begin{aligned}
& \int_{0}^{1}\left[Z^{*}\left(Y^{\prime}+\mathscr{P} Y\right)-\left(-Z^{\prime}+\mathscr{P}^{*} Z\right)^{*} Y\right] d t \\
& \quad=Z(1+) Y(1)-Z(0-) Y(0)+\Phi^{*} \int_{0}^{1} d V(t) Y(t) .
\end{aligned}
$$

If $Z=\supset Y, l Y=Y^{\prime}+\mathscr{P} Y, l^{+} Z=-Z^{\prime}+\mathscr{P}^{*} Z$, then 


$$
\begin{aligned}
\int_{0}^{1}\left[Y^{*}\right. & \left.\left(\beth^{*} l(Y)\right)-\left(l^{+}(\supset Y)\right)^{*} Y\right] d t \\
& =Y^{*}(1) \beth^{*}(1+) Y(1)-Y^{*}(0) \beth^{*}(0-) Y(0)+\Phi^{*} \int_{0}^{1} d V(t) Y(t) .
\end{aligned}
$$

When $\lambda$ is an eigenvalue, the integral vanishes. The right side will entirely vanish if $\supset(1+)=\beth(0-)=0$.

We can actually solve for the transformation 7 . The eigenfunctions $Y$ are given by $Y(t)=Y(t) Y_{0}$. As noted in $\S 4$, Bryan [7] has found that the eigenfunctions $Z$ are given by

$$
Z(t)=-Y^{*}(t)^{-1} \int_{0}^{t} Y^{*}(s) d v^{*}(s) \Phi(Z) .
$$

Letting $Z=\unlhd Y, Y_{0}=\Phi(Z)$, this implies

$$
\supset(t)=-Y^{*}(t)^{-1} \int_{0}^{t} y^{*}(s) d v^{*}(s) y(t)^{-1} .
$$

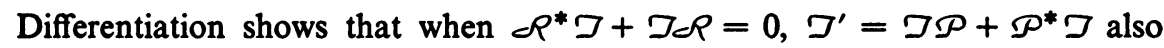
holds for all values of $\lambda . \supset(0-)=0$ is always true, and, when $\lambda$ is an eigenvalue, $\supset(1+)=0$. Inspection shows $d \beth_{s}(t)=-d V^{*}(t) Y(t)^{-1}$. Thus the function $\supset$ has the properties it should. We summarize:

5.2. Theorem. Let the function

$$
\square(t)=-\mathcal{Y}^{*}(t)^{-1} \int_{0}^{t} Y^{*}(s) d v^{*}(s) Y(t)^{-1}
$$

be invariant for all eigenvalues $\lambda$, and satisfy $\mathcal{C} \mathcal{R}^{*} \square+\beth \subset \mathcal{C}=0, \beth^{\prime}=\square \mathcal{P}$ $+\mathscr{P}^{*} \sqsupset, d \beth_{s}=-d V^{*} Y^{-1}, \beth(0-)=0, \beth(1+)=0$. Then the problem

$$
Y^{\prime}+\mathcal{P} Y=\lambda \propto \mathcal{P}, \quad \int_{0}^{1} d v(s) Y(s)=0
$$

is symmetric.

Notice that this theorem is not an if-and-only-if statement. The reason for the omission of the converse statement is the lack of certain algebraic formulae, which were present in the work of Bliss [1] and Reid [20].

Selfadjoint systems.

5.3. Definition. Let of be the Hilbert space generated by the inner product

$$
(Y, Z)=\int_{0}^{1} Z^{*} \unlhd Y d t
$$

where $g=\left(\begin{array}{ll}1 & 0 \\ 0 & 0\end{array}\right)$.

5.4. Definition. Let $D$ be the subspace of 4 consisting of all elements $Y$ satisfying:

(a) The first component $y$ is in $D_{2}$.

(b) There exists an element $F$ in \& such that $l Y=Y^{\prime}+\mathcal{P} Y=\mathcal{C P F}$.

5.5. Definition. We define the differential operator $\mathcal{L}$ by letting $\mathcal{L} Y=F$ for all $Y$ in $D$. 
5.6. Definition. We say that a symmetric system is selfadjoint under $\square(S A T)$ if

(a) $\beth^{*} \mathcal{Q R}=\mathcal{Q} \mathcal{R}^{*} \square$,

(b) $\int_{0}^{1} Y^{*} d \beth_{s} Y=0$

for all $Y$ in $D$.

5.7. Definition. We say that a symmetric system is definite if the only solution to

$$
Y^{\prime}+\mathscr{P} Y=0, \quad \mathcal{R} Y=0, \quad \int_{0}^{1} d V(t) Y(t)=0,
$$

is $Y=0$.

Condition (b) of 5.6 is equivalent to the $\square$ boundary condition derived by Bliss [1] and Reid [20] under endpoint boundary conditions. In the present context it is a much stronger requirement; indeed all its ramifications are not completely understood. It was inadvertently omitted from previous articles [5] and [16].

5.8. Theorem. For a symmetric, definite, SAT system the operator $\beth^{*} \mathcal{L}$ is selfadjoint. 0 is not in the point spectrum of $\mathcal{L}$. $\mathcal{L}^{-1}$ exists and is compact. $\left(\square^{*} \mathcal{L}\right)^{-1}$ is compact and selfadjoint.

Proof. Let $Y_{1}$ and $Y_{2}$ be in $D$ and let $\mathcal{L} Y_{1}=F_{1}, \mathcal{L} Y_{2}=F_{2}$. Then

$$
\begin{aligned}
& \left(\beth^{*} \mathcal{L} Y_{1}, Y_{2}\right)=\int_{0}^{1} Y_{2}^{*} \unlhd \beth^{*}\left(\mathcal{L} Y_{1}\right) d t=\int_{0}^{1} Y_{2}^{*} \beth^{*} \mathcal{L} F_{1} d t \\
& =-\int_{0}^{1} Y_{2}^{*} \beth \mathcal{Q P F _ { 1 }} d t=-\int_{0}^{1} Y_{2}^{*} \beth\left(Y^{\prime}+\mathscr{P} Y\right) d t .
\end{aligned}
$$

Integrating by parts, we find

$$
\begin{aligned}
& \left(\beth^{*} \mathcal{L} Y_{1}, Y_{2}\right)=-\int_{0}^{1} Y_{2}^{*} d \beth_{s} Y_{1}+\int_{0}^{1} Y_{2}^{* \prime} \supset Y_{1} d t+\int_{0}^{1} Y_{2}^{*}\left[\beth^{\prime}-\supset \mathcal{P}\right] Y_{1} d t \\
& =\int_{0}^{1}\left(\Xi^{*}\left[Y_{2}^{\prime}+\mathscr{P} Y_{2}\right]\right)^{*} Y_{1} d t=\int_{0}^{1}\left(\beth^{*} \mathcal{D P F}\right)^{*} Y_{1} d t \\
& =\int_{0}^{1}\left(\beth^{*} \mathcal{L} Y_{2}\right)^{*} \rho Y_{1} d t=\left(Y_{1}, \beth^{*} \mathcal{L} Y_{2}\right) \text {. }
\end{aligned}
$$

This shows $\beth^{*} \mathcal{L}$ is symmetric. Since $\mathcal{L}$ is definite, it possesses an inverse generated by a square integrable Green's function. Therefore since $\square$ is nonsingular, $\beth^{*} \mathcal{L}$ possesses a compact and symmetric, i.e. selfadjoint, inverse. Consequently $\beth^{*} \mathcal{L}$ is selfadjoint.

The eigenfunction expansion. The theory of compact selfadjoint operators in $\&$ immediately implies the following:

5.9. Theorem. For symmetric, definite, SAT systems the spectrum of $\beth^{*} \mathcal{L}$ consists only of real eigenvalues, which accumulate only at $\infty$. For each eigenvalue there exists a finite number of eigenfunctions. If they are $\left\{Y_{j}\right\}_{j=1}^{\infty}$, and if $F$ is in $\mathrm{H}_{\text {, then }}$

$$
F=\sum_{j=1}^{\infty}\left(F, Y_{j}\right) Y_{j},
$$

in the sense of the mean in 4 . 
5.10. Corollary. Let $\left\{y_{j}\right\}_{j=1}^{\infty}$ be the first components of $\left\{Y_{j}\right\}_{j=1}^{\infty}$. If $f$ is in $\mathcal{L}_{n}^{2}(0,1)$, then

$$
f=\sum_{j=1}^{\infty}\left[\int_{0}^{1} y_{j}^{*} f d t\right] y_{j}
$$

in the sense of the mean in $\mathcal{L}_{n}^{2}(0,1)$.

\section{REFERENCES}

1. G. A. Bliss, A boundary value problem for a system of ordinary linear differential equations of the first order, Trans. Amer. Math. Soc. 28 (1926), 561-584.

2. R. P. Boas, Entire functions, Academic Press, New York, 1954. MR 16, 914.

3. R. C. Brown, A determination of the adjoint and Fredholm index of a differential operator with multipoint-integral boundary conditions in $L_{n}^{p}(0,1), 1 \leq p<\infty$, Doctoral Dissertation, Pennsylvania State University, University Park, Pa., 1972.

4. - The existence of the adjoint in linear differential systems with discontinuous boundary conditions, Ann. Mat. Pura Appl. 93 (1972), 269-274.

5. R. C. Brown, G. B. Green and A. M. Krall, Eigenfunction expansions under multipoint-integral boundary conditions, Ann. Mat. Pura Appl. 95 (1973), 231-243.

6. R. C. Brown and A. M. Krall, Adjoints of multipoint-integral boundary value problems. Proc. Amer. Math. Soc. 37 (1973), 213-216.

7. R. N. Bryan, A linear differential system with general linear boundary conditions, J. Differential Equations 5 (1969), 38-48. MR 38 \# 1312.

8. R. Conti, Recent trends in the theory of boundary value problems for ordinary differential equations, Boll. Un. Mat. Ital. (3) 22 (1967), 135-178. MR 36 \# 1734.

9. N. Dinculeanu, Vector measures, Internat. Series of Monographs in Pure and Appl. Math., vol. 95, Pergamon Press, Oxford; VEB Deutscher Verlag der Wissenschaften, Berlin, 1967. MR 34 \#601lb.

10. S. Goldberg, Unbounded linear operators: Theory and applications, McGraw-Hill, New York, 1966. MR 34 \#580.

11. G. B. Green and A. M. Krall, Linear differential systems with infinitely many boundary points, Ann. Mat. Pura Appl. 91 (1972), 53-67.

12. A. Halanay and A. Moro, $A$ boundary value problem and its adjoint, Ann. Mat. Pura Appl. (4) 79 (1968), 399-411. MR 38 \#2371.

13. J. L. Kelley and I. Namioka, Linear topological spaces, University Series in Higher Math., Van Nostrand, Princeton, N. J., 1963. MR 29 \#3851.

14. A. M. Krall, Differential operators and their adjoints under integral and multiple point boundary conditions, J. Differential Equations 4 (1968), 327-336. MR 37 \#6525.

15._, Differential-boundary operators, Trans. Amer. Math. Soc. 154 (1971), 429-458. MR 42 \#6328.

16. Self-adjoint boundary value problems with infinitely many boundary points, Ann. Mat. Pura Appl. 91 (1972), 69-77.

17._, An eigenfunction expansion for a nonselfadjoint interior point boundary value problem, Trans. Amer. Math. Soc. 170 (1972), 137-147.

18. S. Lang, Analysis. II, Addison-Wesley, Reading, Mass., 1969.

19. B. Ja. Levin, Distribution of zeros of entire functions, GITTL, Moscow, 1956; English transl., Transl. Math. Monographs, vol. 5, Amer. Math. Soc., Providence, R. I., 1964. MR 19, 402; 28 \#217.

20. W. T. Reid, A class of two point boundary value problems, Illinois J. Math. 2 (1958), 434-453. MR 20 \#3331.

21. H. L. Royden, Real analysis, Macmillan, New York, 1968. 
22. D. H. Tucker, Boundary value problems for linear differential systems, SIAM J. Appl. Math. 17 (1969), 769-783. MR 40 \#5958.

23. $O$. Vejvoda and M. Tvrdy, Existence of solutions to a linear integro-boundary-differential equation with additional conditions, Ann. Mat. Pura Appl. 89 (1971), 169-216.

24. W. M. Whyburn, Differential systems with general boundary conditions (No. 1, Seminar Rep. in Math.), Univ. California Publ. Math. 2 (1944), 45-61. MR 5, 265.

MATHEMATICS RESEARCH CENTER, UNIVERSITY OF WISCONSIN, MADISON, WISCONSIN 53706

DEPARTMENT OF MATHEMATICS, PENNSYLVANIA STATE UNIVERSITY, UNIVERSITY PARK, PENNSYLVANIA 16802 